\title{
General practitioners' perspectives on primary care consultations for suicidal patients
}

\author{
Pooja Saini PhD Student ${ }^{1}$, Khatidja Chantler $\mathrm{PhD}^{2}$ and Navneet Kapur Professor ${ }^{3}$ \\ ${ }^{1}$ Department of Psychological Sciences, University of Liverpool, Liverpool, UK, ${ }^{2}$ School of Social Work, University of \\ Central Lancashire, Preston, UK and ${ }^{3}$ Centre for Mental Health and Risk, University of Manchester, Manchester, UK
}

Accepted for publication 26 November 2014

\author{
Correspondence \\ Pooja Saini \\ Department of Psychological \\ Sciences \\ University of Liverpool \\ Whelan Building, Room GO4 \\ Brownlow Hill \\ Liverpool L69 3GS, UK \\ E-mail: pooja.saini@liverpool.ac.uk
}

\section{What is known about this topic}

- GPs can play an important role in suicide prevention as a substantial proportion of suicide patients have visited their GPs weeks or months prior to their death.

- Previous studies have been largely descriptive and have focussed on consultation behaviour or clinical characteristics prior to suicide rather than contextual data from consultations.

\section{What this paper adds}

- GPs had difficulty in accurately interpreting patients' talk and behaviour prior to suicide.

- GPs highlighted the need for improved access to secondary care to support patients better and reduce GPs' sense of professional isolation.

- The tensions GPs faced when respecting patient autonomy and fulfilling their professional responsibilities were often in conflict with each other.

\begin{abstract}
Little is known about general practitioners' (GPs') perspectives, management of and interactions with suicidal patients prior to the patient's suicide. The aims of the study were to explore GPs' interpretations of patient communication and treatment in primary care leading up to suicide and to investigate the relationship between GPs and mental health services prior to a patient's suicide. Thirty-nine semistructured interviews with GPs of people who had died by suicide were conducted as part of a retrospective study. Interviews were transcribed verbatim and analysed using a thematic approach. The following themes emerged from GP interviews: (i) GP interpretations of suicide attempts or self-harm; (ii) professional isolation; and (iii) GP responsibilities versus patient autonomy. GPs recruited for the study may have different views from GPs who have never experienced a patient suicide or who have experienced the death of a patient by suicide who was not under the care of specialist services. Our findings may not be representative of the rest of the United Kingdom, although many of the issues identified are likely to apply across services. This study highlighted the following recommendations for future suicide prevention in general practice: increasing GP awareness of suicide-related issues and improving training and risk assessment skills; removing barriers to accessing therapies and treatments needed in primary care; improving liaison and collaboration between services to provide better patient outcomes; and increasing awareness in primary care about why patients may not want treatments offered by focusing on each individual's situational context.
\end{abstract}

Keywords: general practitioner, mental health services, primary care, self-harm, suicide

\section{Introduction}

Research into suicide prevention in primary care is relatively sparse (Schulberg et al. 2004, Mann et al. 2005, Bajaj et al. 2008, Bryan et al. 2009, Kendall \& Wiles 2010, Saini et al. 2010, O'Connor et al. 2013). The identification and management of suicidal ideation in primary care is a central component of suicide prevention policies (Department of Health [DH] 2012). Despite the emphasis placed on primary care, studies have found relatively low levels of assessment of suicide risk among patients treated in this setting (Schulberg et al. 2004, Bryan et al. 2009). Previous work has been unable to determine the balance of benefits and harms of screening for suicide risk in primary care settings, but has recommended that general practitioners (GPs) should be alert to suicidal ideation among patients exhibiting depression and other well-established risk factors such 
as previous suicide attempts, history of self-harm or substance misuse and chronic physical health issues (Schulberg et al. 2004, DH 2012).

Over $90 \%$ of patients who die by suicide have visited their GPs within weeks or months of their death, and the vital role that GPs can play to reduce suicide deaths has been recognised (Luoma et al. 2002, Pearson et al. 2009, Rodi et al. 2010). In a recent large study from the United Kingdom, patients who consulted frequently in primary care were at high risk of suicide, particularly in the 2 or 3 months prior to death (NCISH 2014). Despite evidence that people who die by suicide are more than twice as likely to have seen a primary care provider than a mental health provider prior to their death (Luoma et al. 2002), suicide-related discussions in primary care appear to be rare (Isometsa et al. 1995, Pearson et al. 2009). Patients communicate suicidal intent differently and explicitly inform their GP of life-ending thoughts and plans in $3.3-15 \%$ of primary care consultations prior to suicide (Matthews et al. 1994, Isometsa et al. 1995, Pearson et al. 2009).

Previous research has examined primary care consultations before suicide in those under mental healthcare, but this has been largely limited to descriptive studies of patient characteristics and attendance rates (Luoma et al. 2002, Pearson et al. 2009, Rodi et al. 2010) rather than contextual data from consultations. Some qualitative studies have been carried out, including those which have explored the role of lay persons in suicide prevention (Owens et al. 2011, Owen et al. 2012). However, few have investigated the role that primary care services might play in efforts to reduce the incidence of suicide (Bajaj et al. 2008, Kendall \& Wiles 2010, Saini et al. 2010). To date, much research in primary care has focussed on training GPs to identify, manage and assess suicide risk, particularly in patients with a diagnosis of depression (Milton et al. 1999, McDowell et al. 2011, DH 2012).

The recognition of suicidal ideation in primary care is important. Currently in the United Kingdom, GPs use the patient health questionnaire (PHQ-9) for assessing and monitoring depression (NICE CG23, 2004) and 'biopsychosocial assessments' to assess patients' risk (NICE CG90, 2009). However, the general use of risk scales for suicidal ideation or behaviour is controversial (Quinlivan et al. 2014). Even less clear is what action primary care physicians should take when suicide risk is detected. If risk is high, referral to specialist mental health services is the usual course of action, but there is little official guidance or literature on when or how to refer. Some UKbased guidance suggests that patients should be referred for urgent assessment if they score above particular thresholds on screening instruments (GP Notebook 2011, The IAPT Data Handbook 2011), but the impact of such management decisions is unclear.

General practitioners can play an important role in suicide prevention, but little is known about the specific interactions they have with patients who go on to take their own lives or about GP liaison with mental health services prior to a patient's suicide. Communication and liaison between primary care and mental health service providers is essential for effective referrals and treatment. A lack of communication can result in disruption of care, missed or delayed diagnosis, a loss of data in the referral process, missed appointments and repeated or unnecessary testing (Epstein 1995). Few studies have investigated patient referrals from primary care to mental health services or vice versa (Chew-Graham et al. 2007). One study reported on superficial agreements between the two services on the function of the mental health teams, but the decision-making within the team about referral criteria was inconsistent and did not appear to examine the needs of the referred patient. The referral criteria seemed to concentrate on the needs of the secondary mental healthcare staff (and the pressure they were under) rather than primary care perspectives of the needs of the referred patient. No studies to our knowledge have reported on the communication and liaison between GPs and mental health services for patients who have died by suicide or may be at risk of suicide.

Given these gaps in the literature and lack of qualitative research, our study's purpose was to generate rich narratives from GP interviews to obtain insights that are typically overlooked or more difficult to capture via quantitative means. The specific aims of the study were to explore GPs' interpretations of patient communication and treatment in primary care leading up to suicide and to investigate GPs' views on the relationship between primary care and mental health services prior to a patient's suicide.

\section{Method}

\section{Sample and participants}

Details were obtained from the National Confidential Inquiry into Suicide and Homicide by People with Mental Illness (Inquiry) for a consecutive case series of 50 patients. The patients had died by suicide between 1 January 2005 and 30 June 2007 and had been in contact with mental health services in the 
North West of England. The Inquiry provides a UKwide case series of all suicides by people in contact with mental health services in the year prior to death. The ascertainment procedures are robust and the response rates high (over 95\%) (Appleby et al. 2013). Contact details for GPs of the patients identified from the Inquiry were obtained from patient coroner files or administrative departments of NHS Trusts.

\section{Methods: interviews}

Each patient's named GP was contacted by letter to participate in the study. Of 50 potential GP participants, 39 were interviewed. Nine did not participate as they had retired, left the practice or died, and two GPs changed their minds about participating at the time of the interviews. Thirty-nine semi-structured face-to-face interviews were conducted with GPs between 1 June 2007 and 31 October 2009 and lasted between 20 and 40 minutes. Eleven of the GPs were female, 28 were male and the length of time since qualifying ranged from 8 to 37 years, with an average of 19 years. All interviews took place in the GP surgeries and were recorded with permission and then transcribed verbatim. Where named GPs were unavailable, practices were contacted to nominate a suitable alternative GP who had treated the patient in the year prior to death. All interviewees were sent a participant information sheet and indicated their willingness to participate by completing a consent form. An interview schedule was used to collect data, which was adapted from tools used in previous research (see Pearson et al. 2009, Saini et al. 2010).

General practitioners and the interviewer had access to patient's medical records throughout the interviews. Interviews were carried out to explore GPs' interpretations of patient consultations in primary care by examining (i) the way in which the individual's emotional distress was interpreted by GPs; (ii) GPs' responses to patients; and (iii) the relationships and communication between GPs and mental health services prior to a patient's suicide. GPs were asked for information on their patients who had died by suicide - these included details of physical and mental health problems reported in all consultations and treatment offered in the year before death, specifically the final consultation. GPs were also asked about their concerns for the patient, the factors contributing to death, suicide prevention, their experiences of the local mental health services specifically in the context of patients with suicidal ideation and self-harm. Data analysis was completed following the completion of all 39 interviews.
Ethical approval was granted by the North-west Research Ethics Committee (REC reference: 02/8/74) and individual R\&D approvals were obtained from all the relevant Mental Health Trusts included in the study.

\section{Thematic analysis}

Thematic analysis was selected as an appropriate method for examining the interview data because it provided a way of 'getting close' to the interview material and developing a deeper appreciation of the content. The analysis was conducted by the primary researcher (PS), and by the secondary researcher (KC) who supervised PS. The main themes and codes of interest were determined by using the steps recommended by Braun and Clarke (2006): listening to interview recordings and reading each transcript several times to establish familiarity with the whole interview and generating descriptive codes to represent the main themes. The initial codes of interest were generated systematically across the entire data set and were then grouped into a set of emerging themes. Ongoing analysis refined the specifics and formulated the conceptual name of each theme. The final part of the analysis was the selection of the interview extracts, relating the analysis to the research question and literature. The process of refining and validating the findings was conducted through a collaborative exercise creating iterative feedback loops between the primary and secondary researcher. Further discussion between authors resulted in the identification of themes specifically relevant to the challenges GPs face when treating and managing suicidal patients.

\section{Findings}

General practitioners were the participants in this study and interviews were the principal means of data collection, but patient medical records and secondary care data gave an additional insight into the characteristics of the people who had died and GP-patient consultation prior to death. In terms of patient characteristics, the median age was 44 years (33-56 years), $26(67 \%)$ were male, $20(51 \%)$ were unemployed/long-term sick, 23 (59\%) were single, 17 $(44 \%)$ were living alone, $12(31 \%)$ were inpatients at the time of death, $5(13 \%)$ died by suicide within 3 months of discharge from hospital, $30(77 \%)$ had a history of self-harm, $27(69 \%)$ had a history of substance misuse, $16(41 \%)$ had recent adverse life events and $14(36 \%)$ were non-adherent to medication. Seventeen $(45 \%)$ patients had a primary diagnosis of 
depression and/or anxiety, $4(10 \%)$ schizophrenia, 3 $(7 \%)$ bipolar disorder, 7 (18\%) adjustment/personality disorder, 4 (10\%) alcohol/drug dependency and 4 $(10 \%)$ other disorders such as dementia or no mental health problems. In terms of the method used for suicide, $17(44 \%)$ were by hanging, $13(33 \%)$ by self-poisoning, $3(7 \%)$ by jumping from a height or in front of a moving object, $1(3 \%)$ by drowning, and $5(13 \%)$ from other methods such as cutting, stabbing, firearms, suffocation, burning or electrocution.

All of the patients had consulted their GP at least once (range 1-21 consultations) in a year prior to death - over $30 \%$ had consulted more than eight times. Forty per cent of the patients saw a GP in the month prior to suicide and $92 \%$ within 6 months. Seventy per cent of the GPs interviewed knew the patient well and $21 \%$ of GPs reported that they were concerned for the patient's safety at their final consultation in primary care. The majority of these patients $(88 \%)$ spoke of suicidal ideation at this final consultation.

Following the thematic analysis process, three inter-related themes were conceptualised as reflecting the corpus of this material. The themes illustrate the areas where GPs had difficulty managing and treating suicidal patients. The first theme related to varying interpretations of patients' suicidal talk or behaviour by GPs and was conceptualised as 'GPs' interpretations of suicide attempts or self-harm'. The second theme identified was 'Professional isolation' and relates to treatment availability (or lack of availability) for suicidal patients and the lack of support for GPs. The third theme 'GP responsibilities versus patient autonomy' related to the tension GPs experienced while respecting patient decision-making and attempting to fulfil their duty of care; particularly, when patients did not accept the treatment on offer. Each of these themes is developed below.

\section{GPs' interpretations of suicide attempts or self-harm}

General practitioners reported that the majority of patients communicated their suicidal ideas and intent either directly or indirectly to them prior to the act. These verbal and non-verbal expressions can be seen as 'proverbial red flags that call attention to others of the potential risk of more lethal suicidal behaviour' (Maris et al. 2000, p. 267) and therefore provided key opportunities for suicide prevention measures. Patient disclosure of suicide ideation is an important factor in preventing suicide. However, in this study, GPs often interpreted self-harm or suicidal ideation as attention seeking ('crying wolf') rather than a suicide attempt ('cry for help'), thus affecting the manage- ment and treatment offered to patients. The following examples demonstrate the variation in GPs' interpretation and assessment of the patients who consulted prior to suicide:

Although we put her down as a moderate suicide risk, none of us thought she'd ever do it because she talked about it so much. (GP14)

General practitioners' perceptions of patients who spoke of dying by suicide was that they would never follow this through suicide, perhaps based on the erroneous assumption that 'people who talk about suicide don't do it' (Ramsey et al. 2004 as cited in Owen et al. 2012). This seems puzzling as patients with suicidal ideation have been found to be at higher risk of suicide (Appleby et al. 2012). However, suicide is extremely difficult to predict (Kapur 2000) and is one of the most difficult clinical tasks GPs face.

And he'd done this on numerous occasions. Taken overdoses, not as a suicidal attempt but in an attention seeking, in a mental distress, help me cry for help ... even though he'd had a self-harming history, there was never a disorder. (GP22)

Patients presenting in primary care following numerous overdoses provided potential opportunities for GPs to assess a patient's treatment needs. Some GPs perceived overdosing or other self-harming behaviours as attention seeking and interpreted these behaviours as less worrying than a suicide attempt, thus influencing the treatment offered to aid recovery. Although repeat self-harm is a key predictor for suicide (Appleby et al. 2012), some GPs did not perceive previous self-harm as high risk for completed suicide, particularly where there was no mental health diagnosis. Several factors complicate the interpretation of these communications as, despite the increased risk, the majority of people who have selfharmed do not go on to die by suicide (Gunnell et al. 2004).

This is a chap who when he did eventually become suicidal slashed his wrists... he also jumped out of an upstairs window and had a ruptured spleen. So, two previous serious attempts at suicide and was an inpatient for part of that time and at my prompting, the psychiatrist readmitted him before he was on day or weekend leave from the acute psychiatric ward when he committed suicide in quite a planned way. (GP13)

Even when motives were interpreted as being a 'genuine' suicide attempt, GPs faced difficulties as they were not always in a position to ensure patients got the care that they needed. This GP initiated the inpatient admission and was very concerned for the 
patient's safety following his suicide attempt. He took the action required while the patient was under his care; however, the GP had no involvement regarding the patient's agreed leave. This illustrates the exclusion of GPs in secondary mental healthcare decision-making and the lack of communication between services. The next theme develops this further and highlights that some of the issues relating to GPs' decision-making and assessments may also be related to service availability rather than patient needs.

\section{Professional isolation}

Caring for suicidal patients is demanding, involving intensities of emotional labour, moral dilemmas and repeated losses (Gunaratnam 2011). A GP's role involves acting as a gatekeeper or advocate, particularly when referring patients to community mental health teams (CMHTs). GP decision-making was influenced by what were perceived as rigid criteria set by CMHTs and the lack of mental health resources available to them. From accounts provided by GPs, it appeared that CMHTs set extremely high thresholds for treating suicidal patients, possibly as a way to ration services:

Even if we refer to the CMHT, the patient may not get what the GP asked for if they do not agree ... we need help with patients who are depressed, especially ones who are not making progress on medication ... rapid access needs to be improved for patients GPs are concerned about as it seems unless the patients are in shops buying a machete they're not taken seriously. (GP39)

Repeatedly, GPs were left acting as go-betweens for mental health services and patients with the tension of explaining to patients that interventions were unavailable due to resource constraints. Policy guidelines for suicide prevention (DH 2012) state that GPs should be able to access extra support from CMHTs when required; however, this is not always possible as CMHTs often decline to assess some patients unless they are actively suicidal or they are selfreferred. Difficulties were reported by GPs whose patients had depression and were not improving under medication - these referrals were often returned. They reported being frustrated about the lack of access to interventions for patients who preferred specific treatment options that should have been available to them. Subsequently, GPs were left to manage these patients alone in primary care and reported professional isolation:

Because the patient did not attend his last psychiatric appointment, the psychiatrist discharged him and sent a letter to us stating this. I did not agree with this decision. This is what the psychiatrist wrote: 'I am now discharging him from services and I cannot be held responsible for $\mathrm{Mr} \mathrm{X}^{\prime} \mathrm{s}$ misdoings and lack of responsibility towards his psychological problems'. (GP35)

General practitioners said they were disappointed that patients could be discharged without their involvement, particularly as many of these patients usually required more intervention and follow-up, but subsequently became lost in the system. GPs also reported being lost in a 'referral maze' due to the removal of mental health staff from GP surgeries and less contact with psychiatrists. They reported that they required more support from qualified mental health professionals when managing suicidal patients and suggested that patients presenting with suicidal behaviour needed to be seen by a specialist within 2 weeks as per recommendations for patients with physical health problems. GPs reported that more should be done to ensure mental health treatments were as accessible as treatments for physical health problems. The significant inequalities that continue to exist between physical and mental healthcare have an impact on the number of preventable premature deaths and lower treatment rates for mental health conditions (DH 2010). Similar to previous findings (Saini et al. 2010), GPs reported an underfunding of mental healthcare and said they faced obstacles and delays in the implementation of treatment plans for patients which were out of their control. Long-term treatments were rarely available, and short-term solutions, such as crisis teams, 12-week counselling sessions or graduate mental health workers were used by GPs to manage patients to the best of their ability. Numerous GPs reported being left to manage patients alone in primary care and questioned the removal of community psychiatric nurses (CPNs) from GP practices:

I think it's about time that people turn around and say maybe we should have $\mathrm{CPN}[\mathrm{s}]$ within the surgeries, and they should have always been in the surgeries. The reason why GPs want CPNs in the surgery is because they want support not only for patients but also for themselves. At the end of the day, I am not a psychiatrist, I'm just a GP and I see things and try to do the best I can. (GP24)

In the past, GPs received guidance and advice immediately from psychiatrists and CPNs when concerned about a patient's safety, whereas now they reported that the process could take much longer and leave them feeling professionally isolated. GPs said they were no longer involved in the referral process and were more disengaged from patients' secondary care treatment. There was a negative view about these changes and GPs reported feeling despondent 
about mental health professionals appearing not to share responsibility of patients at risk of suicide. Barriers seemed to have formed in their professional relationships:

We need a faster response with CMHTs that are left around and better follow-up. We do not refer much as a practice. We are very dissatisfied with the service provided. The CMHT do not listen even when there is an urgent referral and I think we need better communication as sometimes we feel like we have to manipulate the system just to get a patient assessed ... we therefore refer to hospital A\&E services for 'on the day' referral as patients will get seen and assessed on the day and they do follow-up as their referrals go to the CMHT. (GP35)

In our study, the majority of GPs reported being aggrieved by the response of mental health services and highlighted their lack of confidence when referring patients who may not be accepted for mental health treatment. In a minority of practices, the relationship between GPs and secondary care was more positive. However, this was more prominent in larger practices where there was regular contact and communication between the services:

The monthly meetings we have with CPNs are very valuable indeed and it helps monitor everybody they're seeing and add anybody else who we have remembered, so that's good. (GP14)

\section{GP responsibilities versus patient autonomy}

While GPs have a duty to preserve life, they also have to respect patient autonomy (a patient's right to make decisions about their own treatment). This is an extremely complex area, and the tension between patient autonomy and preserving life complicates the relationship between the health professional and the suicidal patient (David et al. 2010, Kapur et al. 2010). One dilemma is whether patients should have the right to take their own lives. However, this is clearly not only a GP's dilemma but also one that society as a whole needs to consider. The reason we highlight this tension here is because it often appeared as GP frustration in the interview material and was frequently represented as lack of adherence ('lack of adherence' defined as a failure to take medication or other treatment as prescribed or failure to attend follow-up appointments). Reframing adherence issues in terms of patient autonomy allows for a more complex understanding of GPs' interpretations of patient decision-making. To understand patient autonomy and decision-making, we have schematised key treatment areas where patient autonomy is exercised. Lack of adherence to treatment was frequently influenced by a lack of service availability, mirroring findings from the previous section.

\section{Medication}

Patients exercised autonomy by declining medication as sometimes it was unacceptable to them or they felt that it was not making a discernible difference. In these instances, GPs reported that patients may have preferred other interventions such as counselling or psychotherapy; however, access to these services was limited as waiting lists were up to 18 months long. GPs therefore ended up offering medication as the only available treatment for relieving patients' mental health symptoms:

There are no facilities and it is hard to tell patients about the long waiting lists and I feel that patients are not being taken seriously and that they feel that way too. (GP38)

General practitioners perceived that some patients were specifically declining medication, but were more open to other treatments for mental health issues. The previous quote highlights the lack of choice for patients in primary care which conflicts with the right of patients to make decisions about their medical care. In this context, the only 'choice' patients have is to accept medication to treat their mental health needs/suicide ideation or receive no treatment at all. GPs expressed frustration that patient treatments were not necessarily based on a patient's personal needs or circumstances, but more on which services were available - or unavailable.

\section{Lack of appropriate hospital beds}

He was offered admission but was put on a medical ward because there were no beds in the psychiatric ward. He declined the offer to transfer hospital and they could not section him because he was thought not to be actively suicidal and had no plans to self-harm. (GP21)

The patient in the quote above was offered admission to a hospital further away but refused treatment and while this can be seen as an exercise of choice and agency, we cannot be sure that he would have made the same choice if a bed had been available in a local hospital. The lack of availability of local services may have contributed to his decision-making process and his right to exercise autonomy was clearly in conflict with the professional's 'duty of care' to provide care and treatment. One aspect of the GP's dilemma is whether in such cases, compulsory admission may have been more appropriate, but this too is problematic in the context of patient autonomy because of the stigma and lack of choice associated with it. 


\section{Missing appointments}

General practitioner interviews highlighted the following reasons for why patients missed appointments: first, an active decision not to attend; second, unwell patients sometimes found it difficult to remember to come to appointments; and third, some patients missed appointments for administrative reasons. For example, in some services, patients were expected to phone primary mental health workers to book their appointment for an assessment - if they did not call they were not necessarily followed up and were often categorised as having missed their appointment and referred back to GPs. Fourth, some patients simply did not want to leave their house or were unable to do so for clinical reasons (for example, they were agoraphobic and/or intoxicated). There may be important differences between people missing appointments because they did not want help and those who were keen to seek healthcare but struggled to obtain it or to follow advice. GPs were often left in a predicament to understand the reasons for why a patient missed appointments and how to attend to the needs of such patients.

Patients not attending their appointments for mental health treatment were reported as a cause for concern and in tension with GPs' duty of care:

He was very hard to pin down and was also drug abusing ... He was at risk because he was a poor attendee and he was on depot injections for schizophrenia, but very often it was hard for the CPN to make contact with him, as a lot of the time he was a no show and failed to attend despite the efforts. (GP28)

In our study, numerous GPs reported that they were left in a dilemma when responding to nonattendance; particularly, when the decision not to attend may have been an active one. These patients were often more vulnerable and in need of additional support. GPs recounted being left frustrated and helpless as they were unable to fulfil their professional responsibility. The relationship between GPs and patients, and the responsibilities that existed by virtue of those relationships were particularly important to consider as GPs' professional commitments to care for the patient may have been in conflict with patient autonomy. In this context, autonomy was not merely relational but based on obligation and responsibility.

\section{Clear intent to die}

Some patients discussed openly and honestly with their GP that they wanted to die by suicide and GPs' responses continued to show support and management of the patients to the best of their ability:
When I met him, he was absolutely determined he was going to do it [die by suicide] ... he was one of those people where I was sure he was going to do it and I don't think there was any way round it, I mean that's a bit negative, but he was on high doses of antidepressants which he chose not to take at times. (GP12)

Although some patients did not want any treatment and were intent on taking their own lives, GPs still provided interventions to alleviate patients' symptoms despite their perception that the patient may ultimately die by suicide. GPs fulfilled their duty of care while respecting patient autonomy, for example, when patients chose not to take their medication.

This section highlights how patient autonomy can be context dependent (e.g. on availability of counselling or hospital beds) and cause tension for GPs managing patients at risk of suicide. GPs faced a challenge when implementing treatment management plans as the balance of benefits and risks is such that what is acceptable to one patient may be rejected by another. Therefore, the choice of treatment should be based on the patient's values or preferences, but this was often not the case and was sometimes out of the GP's control. However, of note is that some patients had treatment plans in place and support systems available to them but still took their own lives despite a range of interventions offered. This implies that autonomy is not necessarily related to the number of options available to patients but more related to the individual context of their personal situation.

\section{Discussion}

Our data illustrate the very real struggles experienced by GPs in their attempts to make sense of patient communication of suicidality, to get patients the treatment they need and to respect patient autonomy while fulfilling their professional responsibilities. GPs varied in their views, deeming self-harm or suicidal ideation as attention seeking or 'crying wolf' in some cases but interpreting similar behaviour as a suicide attempt or 'cry for help' in others.

The interview material indicated how GPs were compromised when providing treatment interventions for patients at risk of suicide. The systematic barriers in the implementation of specific mental health treatments impacted upon the delivery of nationally recommended evidence-based treatments (Coulter \& Collins 2011). GPs in this study frequently highlighted the lack of access to specialist staff and this barrier hampered collaborative working relationships. There are examples of joint working and 
formal collaborative care between primary care and CMHTs (Chew-Graham et al. 2007), but we found little evidence for these in our study. Many patients were left under the management of primary care while specialist treatment interventions were sought. Where good relations with mental health teams existed, they were valued, both for easing the referral process and for facilitating further support to address a patient's treatment needs. Importantly, the interview material highlighted the need for better support for GPs managing suicidal patients to reduce their professional isolation.

In the context of patient autonomy, GPs were compromised, as treatments normally comprised medications which were acceptable to some patients but rejected by others, and this was usually out of the GP's control, as were the lack of alternatives to medication. In the healthcare context, understanding autonomy placed an additional responsibility on GPs and an obligation to be diligent and skilful in ascertaining the extent to which choices were autonomous. A professional duty of care demands that one goes beyond respect for autonomy in favour of acknowledging other ethical principles when the context demands it. To consider the meaning of a patient's expressed wish to die, the principles of respect for autonomy and capacity need to be taken into account. It is important for GPs to ask why a request is being made, to examine patients' coping styles, to try to understand the expressive nature of the wish, and consider these in the person's situational and developmental context. Therefore, it appears that a model of autonomy based on freedom to choose does not always meet the requirements of care as articulated by GPs.

The findings of the present study must be interpreted in the context of a number of strengths and limitations. The GPs recruited to the study were a selected group of individuals who had experienced the suicide of a patient who had also been under the care of mental health services. Very little is known about the interaction that GPs have with patients who go on to take their own lives and this study provides findings that contribute to this gap in research. Those who participated might also differ in important ways from those who did not. However, most GPs who were approached to take part in the study did so $(82 \%)$. Our findings may not be representative of the rest of the United Kingdom, although many of the issues we identified are likely to apply across services. It should also be noted that some of our data are now several years old. This is partly a reflection of the time it takes for a coroner's verdict to be finalised and the added ethical and practical complexities of carrying out interview-based studies following a suicide death. Consequently, some of the study findings might not necessarily reflect current clinical practice. It should also be noted that the study is based on people who had contact with mental health services in the year prior to suicide; however, $75 \%$ of people who die by suicide have not had this contact before their death (Appleby et al. 2013). Of course, we could have collected retrospective data on people who died by suicide in the general population, but this would not have allowed us to explore the interaction between primary care and secondary care prior to death which was one of our main aims.

Despite its limitations, our study is one of the few that has taken the direct perspective of primary care providers in relation to consultations prior to a patient's death by suicide. We achieved a reasonable sample size and good response rate. Health and social care policies aim to promote good clinical practice through the assessment and management of patients at risk (NICE 2004, Morgan 2007, Gilbert et al. 2011, DH 2012). Our study adds to existing work and provides valuable new data to inform suicide prevention in primary care. The implications of this research for suicide prevention policy and practice in primary care are highlighted in the following four issues. First, we suggest that there may be benefits in increasing GP awareness of suicide-related issues and improving training and risk assessment skills to ensure patients receive the care that they need. Second, the study highlights the importance of removing barriers to help patients access the therapies and treatments they need in primary care and produce better patient outcomes. Third, it also raises the importance of improving liaison and collaborative working relationships between services to provide GPs with support so that they are not left feeling professionally isolated and managing suicidal patients alone. Lastly, it is clear that GPs have a difficult balancing act when trying to treat suicidal patients who do not want to be treated. To develop more patient-centred care, it is essential to attempt to understand and accommodate patient treatment preferences. This includes understanding why a patient may decline treatment within their situational context. This may help to reduce the tension between patient autonomy and GPs' professional duty of care, contributing to a more patient-centred way of working.

Future work should focus on the barriers to the recognition of risk and provision of care, and build upon the benefits of collaborative care. The treatment of people who have suicidal ideas or behaviour is clearly not just a GP's responsibility but is an issue 
that health services more generally, suicide prevention policies, and society as a whole need to contemplate.

\section{References}

Appleby L., Kapur N., Shaw J. et al. (2012) The National Confidential Inquiry into Suicide and Homicide by People with Mental Illness. Annual report, England, Wales, Scotland and Northern Ireland. Available at: http://www.bbmh. manchester.ac.uk/cmhr/research/centreforsuicideprevention/nci/reports/annual_report_2012.pdf (accessed on 10/11/2013).

Appleby L., Kapur N., Shaw J. et al. (2013) National Confidential Inquiry into Suicide and Homicide by People with Mental Illness. Annual Report, England, Northern Ireland, Scotland and Wales. Available at: http://www.bbmh.manchester. ac.uk/cmhr/centreforsuicideprevention/nci/reports/NCIAnnualReport2013V2.pdf (accessed on 10/11/2013).

Bajaj P., Borreani E., Ghosh P., Methuen C., Patel M. \& Crawford M. (2008) Screening for suicidal thoughts in primary care: the views of patients and general practitioners. Mental Health in Family Medicine 5, 229-235.

Braun V. \& Clarke V. (2006) Using thematic analysis in psychology. Qualitative Research in Psychology 3, 77-101.

Bryan C.J., Corso K.A. \& Neal-Walden T.A. (2009) Managing suicide risk in primary care: practice recommendations for behavioral health consultants. Professional Psychology: Research and Practice 40 (2), 148-155.

Chew-Graham C., Slade M., Montana C., Stewart M. \& Gask L. (2007) A qualitative study of referral to community mental health teams in the UK: exploring the rhetoric and the reality. BMC Health Services Research 7, 117.

Coulter A. \& Collins A. (2011) Making Shared Decision-Making A Reality: 'No Decision about Me without $M e^{\prime}$. The King's Fund, London.

David A.S., Hotopf M. \& Moran P. (2010) Mentally disordered or lacking capacity? Lessons of management for deliberate self-harm. British Medical Journal 341, c4489.

Department of Health (2010) Healthy lives healthy people: our strategy for public health in England. Available at: https:// www.gov.uk/government/uploads/system/uploads / attachment_data/file/216096/dh_127424.pdf (accessed on 10/11/2013).

Department of Health (2012) Preventing suicide in England: a cross-government outcomes strategy to save live. Available at: https://www.gov.uk/government/uploads/system/uplo ads/attachment_data/file/216928/Preventing-Suicide-in-Eng land-A-cross-government-outcomes-strategy-to-save-lives. pdf (accessed on 10/11/2013).

Epstein R.M. (1995) Communication between primary care physicians and consultants. Archives of Family Medicine 4, 403-409.

Gilbert E., Adams A. \& Buckingham C.D. (2011) Examining the relationship between risk assessment and risk management in mental health. Journal of Psychiatric and Mental Health Nursing 18 (10), 862-868.

GP Notebook (2011) Patient health questionnaire (PHQ-9). Available at: http://www.gpnotebook.co.uk/simplepage.cfm? ID=x20090201195702749131 (accessed on 20/9/2014).

Gunaratnam Y. (2011) Cultural vulnerability: a narrative approach to intercultural care. Journal of Qualitative Social Work, 1-15. doi:10.1177/1473325011420323.
Gunnell D., Harbord R., Singleton R., Jenkins R. \& Lewis G. (2004) Factors influencing the development and amelioration of suicidal thoughts in the general population. British Journal of Psychiatry 185, 385-393.

Isometsa E.T., Heikkinen M.E., Marttunen M.J., Henriksson M.M., Aro H.M. \& Lönnqvist J.K. (1995) The last appointment before suicide: is suicide intent communicated? American Journal of Psychiatry 152 (6), 919-922.

Kapur N. (2000) Evaluating risks. Advances in Psychiatric Treatment 6, 399-406.

Kapur N., Clements C. \& Bateman N. (2010) Advance directives and suicidal behaviour. British Medical Journal 341, c4557.

Kendall K. \& Wiles R. (2010) Resisting blame and managing emotion in general practice: the case of patient suicide. Social Science and Medicine 70, 1714-1720.

Luoma J.B., Martin C.E. \& Pearson J.L. (2002) Contact with mental health and primary care providers before suicide: a review of the evidence. American Journal of Psychiatry 159, 909-916.

Mann J.J., Apter A., Bertolote J. et al. (2005) Suicide prevention strategies: a systematic review. Journal of the American Medical Association 294 (16), 2064-2074.

Maris R.W., Berman A.L. \& Silvermann M.M. (2000) Suicide notes and communication. In: R.W. Maris, A.L. Berman \& M.M. Silvermann (Eds) Comprehensive Textbook of Suicidology, p. 267. The Guildford Press, New York.

Matthews K., Milne S. \& Ashcroft G.W. (1994) Role of doctors in the prevention of suicide: the final consultation. British Journal of General Practice 44 (385), 345-348.

McDowell A.K., Lineberry T.W. \& Bostwick J.M. (2011) Practical suicide-risk management for the busy primary care physician. Mayo Clinical Proceedings 86 (8), 792-800.

Milton J., Ferguson B. \& Mills T. (1999) Risk assessment and suicide prevention in primary care. Crisis 20 (4), 171-177.

Morgan J.F. (2007) Giving Up the Culture of Blame: Risk Assessment and Risk Management in Psychiatric Practice. Royal College of Psychiatrists, London.

National Confidential Inquiry into Suicide and Homicide by People with Mental Illness (NCISH) (2014) Suicide in Primary Care in England: 2002-2011. University of Manchester, Manchester.

National Institute for Health and Clinical Excellence (2004) Depression: Management of Depression in Primary and Secondary Care - NICE Guidelines CG23. NHS, UK.

National Institute for Health and Clinical Excellence (2009) Depression in Adults: The Treatment and Management of Depression in Adults - NICE Guidelines CG90. NHS, UK.

O'Connor E., Gaynes B., Burda B., Soh C. \& Whitlock E. (2013) Screening for and treatment of suicide risk relevant to primary care: a systematic review for the U.S. Preventive Services Task Force. Annals of Internal Medicine 158, 741-754.

Owen G., Belem J., Lambert H., Donovan J., Rapport F. \& Owens C. (2012) Suicide communication events: lay interpretation of the communication of suicidal ideation and intent. Social Science and Medicine 75 (2), 419-428.

Owens C., Owen G. \& Belam J. (2011) Recognising and responding to suicidal crisis within family and social networks: qualitative study. British Medical Journal 18 (343), d5801.

Pearson A., Saini P., DaCruz D. et al. (2009) Primary care contact prior to suicide in individuals with mental illness. British Journal of General Practice 59 (568), 826-832. 
Quinlivan L., Cooper J., Steeg S. et al. (2014) Scales for predicting risk following self-harm: an observational study in 32 hospitals in England. British Medical Journal Open 4, e004732.

Ramsey R.F., Tanney B.L., Lang W.A. et al. (2004) Suicide Intervention Handbook, $10^{\text {th }}$ edn. Living Works Education, Calgary. Citied in Owen G., Belem J., Lambert H., Donovan J., Rapport F. \& Owens C. (2012) Suicide communication events: lay interpretation of the communication of suicidal ideation and intent. Social Science and Medicine, 75 (2), 419-428.

Rodi P.M., Roskar S. \& Marusic A. (2010) Suicide victims' last contact with the primary care physician: report from
Slovenia. International Journal of Social Psychiatry 56 (3), 280-287.

Saini P., Windfuhr K., Pearson A. et al. (2010) Suicide prevention in primary care: general practitioners' views on service availability. British Medical Council Research Notes 3, 246.

Schulberg H.C., Hyg M.S., Bruce M.L., Williams J.W. Jr \& Dietrich A.J. (2004) Preventing suicide in primary care patients: the primary care physician's role. General Hospital Psychiatry 26, 337-345.

The IAPT Data Handbook (2011) Guidance on Recording and Monitoring Outcomes to Support Local Evidence-Based Practice. NHS, UK. 